\title{
SUCCESSFUL CQI-BASED PROGRAMS IN A GROUP-MODEL MANAGED CARE SETTING
}

Therapeutic drug intervention involves the replacement of one drug, with another agent of different chemical structure within the same therapeutic category. The replacement agent is considered to be the more appropriate choice based upon clinical, pharmacokinetic, pharmacodynamic, and/or cost considerations. Because pharmaceuticals today may represent as much as $8-12 \%$ of overall healthcare costs, programs such as therapeutic intervention have become a mainstay of pharmacy utilization management in most hospitals and managed care organizations (MCOs). The goal of therapeutic intervention is to ensure that patients experience high-quality pharmaceutical care through sound, economically optimal drug selection.

\section{BACKGROUND}

Harvard Community Health Plan (HCHP) is a mixed-model health-maintenance organization serving more than 650,000 lives in the New England region. In an effort to meet the challenge of providing high-quality, cost-effective pharmaceutical care, HCHP's Medical Groups Division recently entered into a capitated pharmacy arrangement with its pharmacy benefit manager (PBM), PharmaCare. As part of this partnership arrangement, PharmaCare receives from HCHP a set payment per member per month in return for services designed to optimize the quality of pharmaceutical care and to decrease overall pharmacy expenditures.

Through the application of Supplier Quality Management (SQM) principles, various cross-functional work teams were developed to ensure that both organizations achieve maximum individual benefit. A clinical subgroup was formed to develop means of enhancing existing therapeutic substitution and formulary management systems within HCHP's Medical Groups Division. CIMS (Clinical Information Management System), an innovative and successful program, evolved from these efforts. This review describes its development, implementation, and results to date.

\section{DEVELOPMENT}

Optimizing the ability to drive market share of "preferred" drug products within therapeutic categories maximizes an MCOs rebate potential with pharmaceutical manufacturers, increases future contractual capabilities, and lowers overall pharmacy expenditures. Before its capitation arrangement with PharmaCare, HCHP had implemented several educational strategies to achieve these goals. HCHP's unique working relationship with its medical group clinicians aided in increasing market share of preferred formulary products HCHP recognized, however, that existing intervention opportunities had yet to be maximized. Aligning incentives through capitation with PharmaCare was the next logical step.

By benchmarking the activities of other MCOs and PBMs, the clinical SQM work team evaluated available options. An initial pilot program involving network pharmacist telephone intervention was conducted. This program, although moderately successful in converting patients to preferred agents, was deemed a failure because of an overall lack of support on the part of physicians, pharmacists, and patients themselves. Physicians, although willing to support HCHP's conversion efforts, found telephone intervention intrusive and bothersome. Pharmacists, because of time constraints, found the program difficult and burdensome. Patients complained of the lack of adequate notification and communication regarding their switch in medications before refill pickup.

Recognizing the potential to positively impact clinician prescribing and the obvious shortcoming of a telephone intervention program, the clinical work group decided to refocus its efforts. A new intervention approach was conceived that would diminish the burden placed upon both the clinician and phar- macist. In addition, this new approach would keep the patient fully abreast of any authorized medication conversion well in advance of their refill pickup.

\section{IMPLEMENTATION}

This next pilot was designed to use PharmaCare's central pharmacy database and its clinical and administrative staff. HCHP clinicians with the highest number of patients on a nonformulary ACE inhibitor were targeted for inclusion in the pilot. The new intervention methodology would employ written communication to each of these clinicians from HCHP's associate medical director and pharmacy director

As part of this communication, clinicians received educational materials to assist them in understanding both the clinical and financial rationale for the conversion program. This information included a summary of clinical benefits associated with the preferred formulary agent, a dosage conversion chart, and a projected yearly cost savings associated with individual as well as aggregate ACE inhibitor conversions.

To further simplify the process, physician- and patient-specific authorization forms for conversion were created. Each clinician received, along with the communication from HCHP, a set of preprinted authorization 
forms listing each of their patients currently receiving the nonformulary drug product. Authorization forms included all relevant demographic and prescription-specific information necessary for the clinician to convert their patients. Finally, a response card was included in each packet seeking subjective clinician feedback on the program.

Clinicians were given the option of converting patients or maintaining them on their current medication without penalty. If clinicians chose to deny the conversion, they were asked to respond with a clinically valid reason. An approved conversion simply required the completion of appropriate sections of the authorization form. Once complete, forms were signed, dated and returned to PharmaCare in the pre-addressed, stamped envelope provided. The authorization forms were furnished in triplicate so that a copy could be maintained by the clinician for inclusion in each patient's permanent medical record

Authorization forms received at PharmaCare were then reviewed for completeness and accuracy, sorted, and input into a central database. Next, patient-specific letters from HCHP were generated and distributed to all patients whose clinician authorized a conversion. These letters notified the patient that their clinician had reviewed their medical profile and authorized a switch in medication. Attached to this letter was a question and answer sheet designed to clarify the more common concerns the patient might have. Early notification provided the patient the opportunity to contact their clinician, pharmacist, or HCHP if still uncomfortable with the conversion.

Once a predetermined time period had elapsed from the distribution of patient let ters, approved authorization forms were then distributed to network pharmacies for processing. Network pharmacists attended training sessions conducted by PharmaCare before the program implementation. Each received complete packets of conversion materials with every set of authorizations sent. At their convenience, pharmacists were asked to input the newly authorized prescriptions into each patient's prescription drug profile; cancel the previous script; and flag the patient's new medication so that adequate counseling be provided.

Response to the new pilot program was positive. Of more than 400 patients targeted, $82 \%$ were successfully converted to HCHP's preferred ACE inhibitor within a 12-week time period, for an annualized cosl savings in excess of $\$ 50,000$. The success of the pilot generated universal support from medical directors and administrators, thus warranting further expansion of the program.

\section{EXPANSION}

Since the ACE inhibitor pilot, additional interventions have been successfully launched in several different therapeutic categories, including $\mathrm{H}^{2}$ antagonists, HMG $\mathrm{CoA}$ reductase inhibitors, dihydopyridine calcium blockers, and triphasic oral contraceptives. To date, these programs have generated an estimated $\$ 700,000$ in pharmacy savings.

With each intervention effort, HCHP and PhamaCare have strived to refine the process to ensure its clinical quality and financial success. Appropriate monitoring systems have been set up to verify that preferred formulary alternatives are indeed providing the therapeutic benefit intended. Authorization forms have been revised to capture clinical diagnoses. Tracking systems have been developed to monitor switchback rates and clinical rationale for re-conversion. And finally, clinical and administrative resources have been expanded to streamline operational activities and broaden clinical support mechanisms. Given the success thus far experienced in the area of therapeutic intervention, the SQM clinical work team is currently developing means of expanding the CIMS process to alert clinicians of patient noncompliance and also to further enhance the retrospective drug use review programs currently in place at HCHP. In doing so, HCHP and PharmaCare hope to bring additional clinical value to the CIMS program and generate further clinician support. The ultimate goal of the CIMS program is to assist the providers in the ability to better manage the overall healthcare of their patients in the most cost-effective manner possible.

\section{CONCLUSION}

The evolution of the healthcare delivery system has created the need for unique, cooperative alliances to ensure the success of a managed care pharmacy benefit program. The capitated arrangement between HCHP and PharmaCare is a prime example of how providers and suppliers can come together to gain mutually beneficial results. Aligned incentives can increase the ability to maximize the value of pharmaceutical care, improve patient outcomes, and lower overall healthcare costs.

The PharmaCare CIMS program has created a unique communication link between the managed care organization, its clinicians, network pharmacists, and patients. Its success indicates that active intervention and cooperation between an MCO and PBM, when supported by a wellsiruciured formulary system, can indeed produce substantial value. The application of supplier quality management principals provides the foundation to overcome common barriers and build the trust, integrity, and cooperation necessary to create a "win-win" situation for everybody involved.

\footnotetext{
David Calabrese, B.Sc., R.Ph. Clinical Pharmacy Coordinator Harvard Community Health Plan Medical Groups Division 10 Brookline Place West, 2nd Floor Brookline, MA 02146
} 